Z. Klin. Chem. Klin. Biochem.

11. Jg. 1973, S. $20-23$

\title{
Zum Zusammenhang zwischen Proteinsynthesehemmung und Induktion mikrosomaler Enzyme durch Barbital
}

\author{
VON H. KRÖNER \\ Institut für Pbysiologische Chemie der Universität Düsseldorf
}

(Eingegangen am 13. Juni/29. September 1972)

Barbital-Natrium bewirkt eine Hemmung der Protein- und RNA-Synthese in der Rattenleber, die zum Teil auf der alkalischen Reaktion der Injektionslösung und zum Teil auf einem Abfall der Körpertemperatur beruht. Es wird untersucht, ob durch diese Faktoren die spätere Induktion mikrosomaler Enzyme beeinflußt wird. Als Maß dienen die Hexobarbitalschlafzeit, die Dauer der Zoxazolaminlähmung und die Geschwindigkeit der Metabolisierung von Hexobarbital und Dimethylaminophenazon durch Lebermikrosomen in vitro. Aufgrund der Ergebnisse muß ein Kausalzusammenhang zwischen akuter Hemmung der Proteinsynthese und späterer Enzyminduktion abgelehnt werden.

\section{The relationship between the inbibition of protein synthesis and the induction of microsomal encymes by barbital}

Sodium barbital causes an inhibition of protein and RNA synthesis in rat liver, which is due partly to the alkaline reaction of the injection solution and partly to a fall in body temperature. Experiments were undertaken to determine whether these factors influence the later induction of microsomal enzymes. The criteria used were the hexobarbital sleeping time, the duration of Zoxazolamine paralysis and the rate of metabolism; of hexobarbital and dimethylaminophenazone by liver microsomes in vitro. On the basis of the results, no causal relationship can be claimed between the acute inhibition of protein synthesis and the later enzyme induction.

Während das Phänomen der Enzyminduktion bei Mikroorganismen in den letzten Jahren zum Teil bis ins Detail aufgeklärt werden konnte (1), ist das, was bei höheren Organismen mit diesem Terminus bezeichnet wird, noch reichlich undurchsichtig. Unsere Vorstellungen über die Induktion durch Hormone beruhen noch weitgehend auf Analogieschlüssen zu Abläufen bei Bakterien.

Fast noch unbefriedigender erscheinen die Aspekte, die sich ergeben, wenn man die "Induktion" mikrosomaler, Drogen abbauender Enzyme in der Leber von Säugetieren betrachtet. Die Zahl der Substanzen unterschiedlichster chemischer Struktur ist nicht abzusehen (2), die im Tierexperiment meist innerhalb weniger Tage über eine gesteigerte RNA- und Proteinsynthese $(3,4,5)$ zu einer Aktivitätszunahme jener mikrosomalen Enzyme führt. Die Aufklärung dieses Induktionsmechanismus erscheint besonders deshalb schwierig, weil irgendwelche Gemeinsamkeiten der induzierenden Pharmaka kaum zu erkennen sind, es sei denn, man hält die relativ gute Fettlöslichkeit der meisten Substanzen für ausreichend.

Die früher beschriebene akute Hemmung der Proteinund RNA-Synthese durch Barbital-Natrium (6) und die Beobachtung anderer Autoren $(7,8,9)$, daß Substanzen, die primär die Proteinsynthese hemmen, sekundär eine Induktion hervorrufen, ließen den Gedanken an einen Zusammenhang zwischen primärem Hemmeffekt und sekundärer Auslösung einer Induktion aufkommen (10), wobei ein solcher Kausalzusammenhang nicht auf Barbitaleffekte beschränkt sein müßte, sondern auch zur Erklärung der Enzyminduktion durch andere Pharmaka beitragen könnte.
Die akute Hemmung der Proteinsynthese durch Barbital-Natrium beruht zum Teil auf einem Abfall der Körpertemperatur (11), zum Teil auf der Alkalinität des Präparates (12). Werden diese beiden Effekte durch Neutralisation der Injektionslösung und Unterbringung der Tiere bei erhöhter Raumtemperatur während des Versuches ausgeschaltet, so ist damit auch weitgehend die Hemmung der Proteinsynthese durch Barbital aufgehoben. Ein Vergleich der Enzyminduktion bei zwei Kollektiven, von welchen bei einem die akute Proteinsynthesehemmung ausgeschaltet wurde, soll die Frage nach dem Zusammenhang beider Phänomene beantworten. Das Ausmaß der Induktion mikrosomaler Enzyme wird in vivo erfaßt durch die Hexobarbitalschlafzeit und die Dauer der Zoxazolaminlähmung. In vitro wird die Abbaurate von Hexobarbital und Dimethylaminophenazon durch Lebermikrosomen gemessen.

\section{Methodik}

Die Untersuchungen wurden an weiblichen Wistar-Ratten der Firma Brünger, Bokel, durchgeführt, mit Ausnahme einer Versuchsserie, bei der männliche Versuchstiere verwendet wurden. Die Tiere erhielten Standardfutter der Firma Höveler, Langenfeld-Immigrath, und Wasser ad libitum. Auf ein möglichst einheitliches Körpergewicht in den Versuchsserien wurde besonderer Wert gelegt.

Die Versuchstiere wurden jeweils in drei Kollektive unterteilt: Kollektiv $\mathrm{A}$ erhielt an drei aufeinanderfolgenden Tagen eine i. p. Injektion von Barbital-Natrium (Veronal, Firma Merck) $150 \mathrm{mg} / \mathrm{kg}$ Ratte. Von der Substanz wurden $15 \mathrm{~g} / \mathrm{l}$ in physiol. $\mathrm{NaCl}-\mathrm{L}$ ösung gelöst. Während dieser Behandlung wurden die Tiere bei einer Raumtemperatur von etwa $20^{\circ} \mathrm{C}$ gehalten.

Kollektiv B ethielt ebenfalls eine i. p. Injektion von BarbitalNatrium in gleicher Dosierung, jedoch.wurde die zu injizierende Lösung mit verdünnter Salzsäure auf $\mathrm{pH}$ 7,2 eingestellt. Während 
der dreitägigen Behandlung wurden diese Tiere bei einer Raumtemperatur von $32^{\circ} \mathrm{C}$ gehalten.

Das dritte Kollektiv, die Kontrolltiere, erhiclten entsprechende Mengen physiol. NaCl-Lösung i. p. injiziert und wurden bei Raumtemperatur $\left(20^{\circ} \mathrm{C}\right)$ gehalten.

Zur Bestimmung der Hexobarbital-Schlafzeit (13) exhielten männliche Tiere (mittleres Tiergewicht $200 \mathrm{~g}$ ) eine i. p. Injektion von $200 \mathrm{mg}$ Hexobarbital-Natrium (Evipan-Natrium Firma Bayer) pro $\mathrm{kg}$, weibliche Tiere (mittleres Körpergewicht $180 \mathrm{~g}$ ) bekamen eine Dosis ron $100 \mathrm{mg} / \mathrm{kg}$ Körpergewicht. Während der Schlafzeit waren alle Tiere in einem auf $32^{\circ} \mathrm{C}$ temperierten Raum untergebracht.

Die Dauer der Zoxazolamin-Lähmung (14) wurde bei weiblichen Ratten (mittleres Tiergewicht $200 \mathrm{~g}$ ) nach i. p. Injektion von $100 \mathrm{mg}$ 2-Amino-5-chlorbenzoxazol (Firma Schuchardt) je kg Körpergewicht gemessen. Auch dieser Versuch wurde bei $32^{\circ} \mathrm{C}$ Raumtemperatur durchgeführt.

Zur Messung des Arzneimittelabbaues in vitro wurden weibliche Ratten mit einem mittleren Körpergewicht von $110 \mathrm{~g}$ nach entsprechender dreitägiger Vorbehandlung mit Äther narkotisiert, die Lebern mit eiskalter $0,25 \mathrm{~mol} / 1$ Saccharoselösung von der Pfortader in situ durchspült, die Lebern entnommen und im PotTer-Elvehjem-Homogenisator mit vier Teilen kalter $0,25 \mathrm{~mol} / 1$ Saccharoselösung homogenisiert. Nach Abzentrifugieren der Kerne und Mitochondrien, $10 \mathrm{~min} 800 \mathrm{~g}$ und $20 \mathrm{~min} 15000 \mathrm{~g}$, wurde in der Ultrazentrifuge Spinco L 2, Firma Beckman, in ciner Stunde be: $105000 \mathrm{~g}$ die Mikrosomenfraktion gewonnen. Diesc Mikrosomen wurden mit Hexobarbital (Evipan-Natrium, Firma Bayer) oder Dimethylaminophenazon (4-Dimethylamino2,3-dimethyl-1-phenylpyrazolon (Firma Merck) als Substrat und einem NADPH regenerierenden System nach CONNEY (14) inkubiert (Reagenzien der Firma Boehringer). Nach einstündiger Inkubation im Schüttelthermostat bei $37^{\circ} \mathrm{C}$ unter Luftzutritt wurde die Abnahme von Hexobarbital (15) bzw. das aus Dimethylaminophenazon entstandene 4-Aminoantipyrin (16) gemessen. Eichsubstanz für diese Messung war 1-Phenyl-2,3-dimethyl-4aminopyrazolon-(5) der Firma Merck. Die Bestimmung des Leberglykogens erfolgte nach einer modifizierten PFLüGERMethode mit dem Anthronreagenz (17). Die Bestimmung des Leberproteingehaltes wurde mit dem Biuretreagenz nach WEICHSELBAUM (18) durchgeführt.

\section{Ergebnisse}

Die Narkosedauer nach einer einmaligen Injektjon von Hexobarbital, $200 \mathrm{mg} / \mathrm{kg}$, ist bei erwachsenen männlichen Ratten im Mittel um 40\% verkürzt, wenn die Tiere drei Tage lang mit Barbital-Natrium, $150 \mathrm{mg} / \mathrm{kg} / \mathrm{Tag}$ vorbehandelt wurden (Tab. 1). Dieser Unterschied ist statistisch signifikant, unabhängig davon, ob die Barbitallösung auf einen physiologischen $\mathrm{pH}$-Wert gebracht wird oder nicht und unabhängig davon, ob die Tiere während der Vorbehandlung bei einer Raumtemperatur von $20^{\circ} \mathrm{C}$ oder $32^{\circ} \mathrm{C}$ gehalten wurden.

Weibliche Ratten schlafen nach einer halb so großen Hexobarbitaldosis - $100 \mathrm{mg} / \mathrm{kg}$ i. p. - im Mittel annähernd doppelt so lange wie männliche Tiere mit der doppelten Dosis. Die Schlafzeit wird bei weiblichen Tieren durch Vorbehandlung mit Barbital-Natrium stärker verkürzt als bei männlichen Ratten. Werden die weiblichen Tiere mit der alkalischen Barbital-NatriumLösung vorbehandelt und während der Vorbehandlung bei $20^{\circ} \mathrm{C}$ Raumtemperatur gehalten, so ist die Narkosedauer fast auf $1 / 3$ verkürzt. Wird die Barbital-NatriumLösung dagegen neutralisiert und die Tiere während der Vorbehandlung bei $32^{\circ} \mathrm{C}$ Raumtemperatur gehalten, so wird die Narkosedauer nur auf etwa die Hälfte verkürzt. Diese Differenz der Schlafzeit der beiden Kollektive mit unterschiedlicher Vorbehandlung ist statistisch nicht signifikant $(p \sim 0,1)$, die Schlafzeitverkürzung gegenüber dem Kontrollkollektiv ist in beiden Fällen statistisch signifikant (Tab. 1).

Die Dauer einer Zoxazolamin-Lähmung wird durch die Vorbehandlung prinzipiell ähnlich beeinflußt wie die Hexobarbitalschlafzeit. Beide mit Barbital-Natrium vorbehandelten Kollektive unterscheiden sich durch eine signifikant kürzere Lähmungsdauer von den unbehandelten Kontrollen. Bei den Tieren des Kollektives A (mit alkalischer Barbital-Natrium-Lösung bei Raumtemperatur $\left(20^{\circ} \mathrm{C}\right)$ behandelt) war die Verkürzung zwar ausgeprägter, doch bestand kein signifikanter Unterschied zum Kollektiv B (mit neutralisiertem Barbital-Natrium bei $32^{\circ} \mathrm{C}$ Raumtemperatur behandelt) (Tab. 1).

Die in vitro gemessene Aktivität mikrosomaler, Arzneimittel abbauender Enzyme steigt während der dreitägigen Behandlung mit Barbital-Natrium signifikant um etwa $100 \%$ (Abb. 2). Sowohl der Abbau von Hexobarbital als auch die Demethylierung von Dimethylaminophenazon sind entsprechend beschleunigt. Bei der letzteren Versuchsserie erscheint die Behandlung mit neutralisierter Barbital-Natrium-Lösung bei $32^{\circ} \mathrm{C}$ Raumtemperatur einen etwas größeren Effekt $z u$ haben, doch ergeben sich auch hier keine statistisch signifikanten Unterschiede zwischen den beiden unterschiedlich vorbehandelten Kollektiven.

Tab. 1

Einfluß einer dreitägigen Vorbehandlung mit Barbital auf die Hexobarbitalschlafzeit und die Zoxazolaminlähmung am 4. Tag. Angabe der Zeiten in Minuten \pm Standardabweichung

$\mathrm{n}=$ Zahl der Versuchstiere, $\mathrm{p}=$ Irrtumswahrscheinlichkeit gegenüber Kontrollen

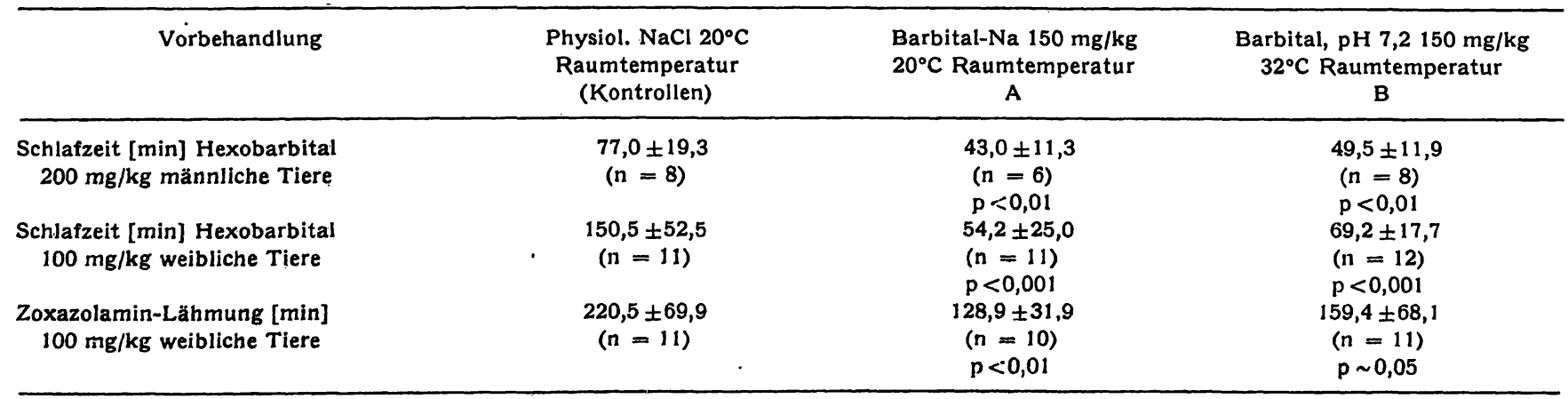


Tab. 2

Einfluß einer dreitägigen Vorbehandlung mit Barbital in vivo auf die Metabolisierung von Hexobarbital und Dimethylaminophenazon in vitro durch Rattenlebermikrosomen. Angaben in $\mu \mathrm{mol}$ Substratumsatz pro $\mathrm{g}$ Frischleber und Stunde $\mathrm{n}=$ Zahl der Messungen, $\mathrm{p}=$ Irrtumswahrscheinlichkeit gegenüber Kontrollen. Einzelheiten siehe Methodik

\begin{tabular}{|c|c|c|c|}
\hline Vorbehandlung & $\begin{array}{c}\text { Physiol. NaCl } \\
20^{\circ} \mathrm{C} \text { Raumtemperatur } \\
\text { (Kontrollen) }\end{array}$ & $\begin{array}{l}\text { Barbital-Na } 150 \mathrm{mg} / \mathrm{kg} \\
20^{\circ} \mathrm{C} \text { Raumtemperatur } \\
\text { A }\end{array}$ & $\begin{array}{l}\text { Barbital, pH 7,2 } 150 \mathrm{mg} / \mathrm{kg} \\
32^{\circ} \mathrm{C} \text { Raumtemperatur } \\
\text { B }\end{array}$ \\
\hline $\begin{array}{l}\text { Abbau von Hexobarbital } \\
{[\mu \mathrm{mol} / \mathrm{h} \cdot \mathrm{g}]}\end{array}$ & $\begin{array}{l}0,27 \pm 0,07 \\
(n=6)\end{array}$ & $\begin{array}{c}0,63 \pm 0,15 \\
(n=6) \\
p<0,001\end{array}$ & $\begin{array}{l}0,60 \pm 0,14 \\
,(n=6) \\
p<0,001\end{array}$ \\
\hline $\begin{array}{l}\text { Demethylierung von Dimethyl- } \\
\text { aminophenazon }[\mu \mathrm{mol} / \mathrm{h} \cdot \mathrm{g}]\end{array}$ & $\begin{array}{c}0,036 \pm 0,018 \\
(n=9)\end{array}$ & $\begin{array}{c}0,062 \pm 0,030 \\
(n=9) \\
p<0,05\end{array}$ & $\begin{array}{c}0,077 \pm 0,022 \\
(n=8) \\
p<0,001\end{array}$ \\
\hline
\end{tabular}

Tab. 3

Einfluß einer dreitägigen Vorbehandlung mit Barbital auf das Leberfrischgewicht, das Trockengewicht, den Glykogen- und Proteingehalt der Leber gefütterter Ratten mit einem mittleren Körpergewicht von $110 \mathrm{~g}$ Mittelwert von $\mathrm{n}$ Bestimmungen \pm Standardabweichung, $\mathrm{p}=$ Irrtumswahrscheinlichkeit gegenüber Kontrollen

\begin{tabular}{|c|c|c|c|}
\hline Vorbehandlung & $\begin{array}{c}\text { Physiol. NaCl } \\
20^{\circ} \mathrm{C} \text { Raumtemperatur } \\
\text { (Kontrollen) }\end{array}$ & $\begin{array}{l}\text { Barbital-Na } 150 \mathrm{mg} / \mathrm{kg} \\
20^{\circ} \mathrm{C} \text { Raumtemperatur } \\
\text { A }\end{array}$ & $\begin{array}{c}\text { Barbital, pH 7,2 } 150 \mathrm{mg} / \mathrm{kg} \\
32^{\circ} \mathrm{C} \text { Raumtemperatur } \\
\text { B }\end{array}$ \\
\hline $\begin{array}{l}\text { Leberfrischgewicht gefütterter } \\
\text { Ratten [g] }\end{array}$ & $\begin{array}{l}6,12 \pm 1,26 \\
(n=12)\end{array}$ & $\begin{array}{c}6,53 \pm 0,85 \\
(\mathrm{n} \equiv 11)\end{array}$ & $\begin{array}{c}5,80 \pm 1,04 \\
(n=12)\end{array}$ \\
\hline $\begin{array}{l}\text { Glykogengehalt } \\
\text { [mg/g Leberfrischgewicht] }\end{array}$ & $\begin{array}{l}64,0 \pm 12,0 \\
(n=6)\end{array}$ & $\begin{array}{l}48,7 \pm 13,1 \\
(n=6)\end{array}$ & $\begin{array}{l}40,9 \pm 19,1 \\
(n=6) \\
p<0,05\end{array}$ \\
\hline $\begin{array}{l}\text { Proteingehalt } \quad \vdots \vdots \\
\text { [mg/g Leberfrischgewicht] }\end{array}$ & $\begin{aligned} & 283 \pm 16 \\
&(n=6) \mid\end{aligned}$ & $\begin{array}{c}291 \pm 34 \\
(n=6)\end{array}$ & $\begin{array}{r}288 \pm 19 \\
(n=6)\end{array}$ \\
\hline $\begin{array}{l}\text { Lebertrockengewicht } \\
\text { [\% vom Frischgewicht] }\end{array}$ & $\begin{array}{c}30,8 \pm 0,8 \\
(n=6)\end{array}$ & $\begin{array}{c}30,0 \pm 2,2 \\
(n=6)\end{array}$ & $\begin{array}{c}30,5 \pm 0,8 \\
(\mathrm{n}=6)\end{array}$ \\
\hline
\end{tabular}

In Tabelle 3 sind einige allgemeine, die Leber der Versuchstiere betreffende Parameter zusammengestellt. Eine statistisch signifikante Differenz ergibt sich nur zwischen dem Glykogengehalt der mit neutralisierter BarbitalNatrium-Lösung bei $32^{\circ} \mathrm{C}$ behandelten Ratten und dem Glykogengehalt der Kontrolltiere. Es sei ferner hingewiesen auf die unterschiedliche Entwicklung des Leberfrischgewichtes bei den beiden unterschiedlich behandelten Kollektiven: im einen Fall eine geringe $\mathrm{Zu}-$ nahme, im anderen eine geringe Abnahme gegenüber den Kontrollen, wobei betont werden muß, daß diese Differenzen statistisch nicht zu sichern waren.

\section{Diskussion}

Für den Zusammenhang zwischen einer akuten Hemmung von Protein- und RNA-Synthese und einer anschließenden Enzyminduktion bestehen prinzipiell zwei Möglichkeiten. Zum einen ist es charakteristisch für Induktionen, daß sie sich durch Hemmung der Proteinoder RNA-Synthese ganz oder teilweise blockieren lassen (19). Das trifft auch für Barbital-Natrium zu, indem es sowohl die Induktion der Tryptophanpyrrolase (EC 1.13.1.12) durch Cortisol als auch die Induktion der Serindehydratase (EC 4.2.1.16) durch Aminosäuren hemmt (20). Zum anderen wurde einleitend schon auf die Möglichkeit hingewiesen, daß die „Induktion“ eine Folge des akuten Hemmeffektes sein könnte $(7,8,9)$. $\mathrm{Zu}$ denken wäre in diesem Zusammenhang zunächst an die Atmungshemmung oder die Entkoppelung der oxidativen Phosphorylierung durch viele induzierende Pharmaka (10). Darüber hinaus ist von vielen dieser
Substanzen eine biphasische Wirkung beschrieben, deren primäre Hemmphase aber auf einer direkten Wirkung dieser Substanzen auf die mikrosomalen Enzyme beruhen dürfte $(21,22,23,24,25)$.

Die zunächst durchgeführten Versuche in vivo zum Zusammenhang zwischen Hemmeffekt und Induktionswirkung von Barbital lassen an die zweite Möglichkeit denken: Bei weitgehender Aufhebung der Barbitalhemmung durch Neutralisation der Injektionslösung und Unterbringung der Tiere bei erhöhter Raumtemperatur erscheint die Induktionswirkung geringer. Die Verkürzung der Hexobarbitalschlafzeit bzw. der Zoxazolaminlähmung ist nicht so ausgeprägt. Diese Differenzen sind aber nicht statistisch gesichert. Die weitere Überprüfung durch Messung von Enzymaktivitäten in vitro macht einen Zusammenhang zwischen Proteinsynthesehemmung und Induktionswirkung von Barbital ganz unwahrscheinlich. Die Aktivität der mikrosomalen Enzyme ist bei Aufhebung des primären Hemmeffektes sicher nicht geringer. Nưn sind diese Enzymaktivitäten auf das Lebergewicht bezogen. Berücksichtigt man, daß dieses Lebergewicht (Tab. 3) bei dem Kollektiv B (mit neutralisierter Barbitallösung behandelte, bẹ erhöhter Raumtemperatur untergebrachte Tiere) geringer ist als bei den anderen Tieren, und bezieht die Enzymaktivität auf das Tiergewicht, so erhält man das gleiche Ergebnis wie bei den in vivo Versuchen. $\mathrm{Da}$ aber auch die Differenzen im Lebergewicht nicht statistisch zu sichern sind, muß ein Kausalzusammenhang zwischen akuter Proteinsynthesehemmung und sekundärer Induktion mikrosomaler Enzyme durch Barbital abgelehnt werden. 


\section{Literatur}

1. Müller-Hill, B. (1971), Angew. Chem. 83, 195-207. - 2. Conney, A. H. (1967), Pharmacol. Rev. 19, 317-366. - 3. Kunz, W., Schaude, G., Schimassex, H., Schmid, W. \& Siess, M. (1966), Proceedings of the European Society for the study of drug toxicity Vol. VII, 138-153. - 4. KaTO, R. JONDORF, W. R., Loeb, L. A., Ben, T. \& Gelbon, H. V. (1966), Mol. Pharmacol. 2, 171-186. - 5. Gelborn, H. V. (1965), Exp. Med. Surg. 23, 85-103. - 6. KRÖNER, H. GUTENBERGER, B., HOLlmanN, S. \& Staib, W. (1969), diese Z. 7, 8-13. - 7. Jondorf, W. R., Simon, D. C. \& Avnimeleck, M. (1966), Biochem. Biophys. Res. Comm. 22; 644-649. - 8. Rosen, F., Raina, P. N., Mtlholland, R. J. \& Nrchor, CH. A. (1964), Science (Washington) 146, 661-663. 9. Fiala, S. \& Fiala, E. S. (1967), Science 157, 1591. - 10. Hollmand, S. \& Neubaur, J. (1967), Hoppe-Scyler's Z. Physiol. Chem. 348, 877-881. - 11. KRöNER, H. \& StaIB, W. (1970), diese Z. 8, 41-44. - 12. KRöNER, H., RudORFF, K.-H. \& StAIB, W. (1970), diese Z. 8, 456-468. - 13. Remmer, H. (1963), Naunyn-Schmiedebergs Arch. Pharmacol. Exp. Pathol. 244, 311-333. - 14. Conney, A. H., Davison, C., Gaster, R. \&
Burns, J. J. (1960), J. Pharmacol. Exp. Ther. 130, 1-8. - 15. Brodie, B. B., Burns, J. J., MArk, L. C., Lief, P. A., Bernstein, E. \& PAPper, E. M. (1953), J. Pharmacol. Exp. Ther. 109, 26-34. 16. Brodie, B. B. \& Axelrod, J. (1950), J. Pharmacol. Exp. Ther. 99, 171-184. - 17. Hassid, W. Z. \& Abraham, S. (1957), in Methods in Enzymology (Colowick, S. P. \& Kaplan, N. O. ed.) Vol. III, S. 34-50, Academic Press New York. - 18. Weichselbaum, T. E. (1946), Amer. J. Clin. Pathol. 16, 40-49. 19. Neubert, D.' (1966), Internist 7, 435-454. - 20. KRÖNER, H., Bojar, H.-E., Hollainn, S. \& Staib, W. (1970), diese Z. 8, 45-48. - 21. BRAzDA, F. G. \& BAuCuM, R. (1961), J. Pharmacol. Exp. Ther. 132, 295-298. - 22. Remier, H. (1962), Proc. 1st Int. Pharmacol. Meeting Stockholm (Brodie, B. B. ed.) Vol. 6, p. 235-256, Mac Millan, New York. - 23. Serrone, D. M. \& Fujimoto, J. M. (1962), Biochem. Pharmacol. 11, 609-615. - 24. RümKe, C. L. (1963), Schmiedebergs Arch. Pharmacol. Exp. Pathol. 244, 519-530. - 25. Kato, R., Chíresara, E. \& Vassanelit, P. (1964), Biochem. Pharmacol. 13, 69-83.

Privatdozent Dr. H. Kröner 4000 Düsseldorf

Moorenstr. 5 